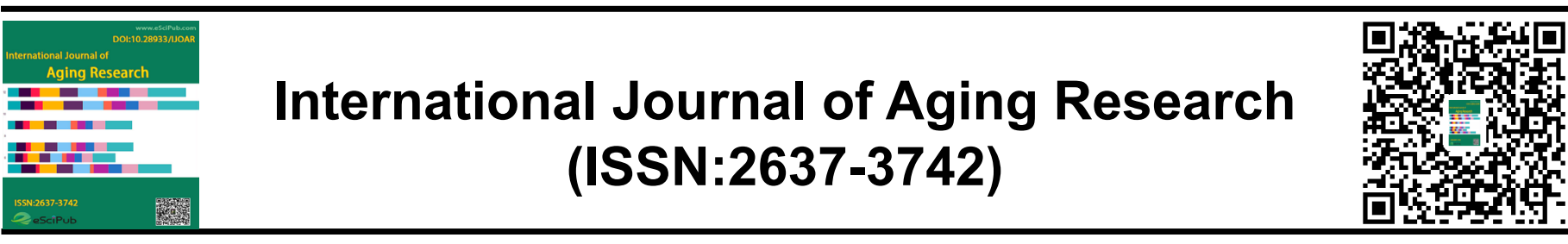

\title{
Development of Estimate Formulas for Waist Circumference Using Body Mass Index and Limb Circumferences in Hospitalized Older
}

\section{Adults}

\section{Daisuke Takagi, $\mathrm{PhD}^{1}$, Masatoshi Kageyama ${ }^{2,3}$, Kenta Yamamoto ${ }^{4}$, Hiroshi Matsumoto, $\mathrm{MD}^{5}$}

${ }^{1}$ Department of Physical Therapy, Health Science University; ${ }^{2}$ Long-Term Care Health Facilities Sunrise Ohama; ${ }^{3}$ Graduate School, Hamamatsu University School of Medicine, Cooperative Major in Medical Photonics (Doctoral Course); ${ }^{4}$ Department of Rehabilitation, Toyoda Eisei Hospital; ${ }^{5}$ Department of Orthopedic surgery, Toyoda Eisei Hospital

\section{ABSTRACT}

Background: Little research has been conducted on the estimate formulas for waist circumference using body mass index and limb circumferences in hospitalized older adults. Thus, we conducted the present study to develop estimate formulas of waist circumference using body mass index and limb circumferences in hospitalized older adults. Methods: Forty hospitalized older patients were recruited in this cross-sectional study. We measured waist circumference, body mass index, upper arm circumference, forearm circumference, thigh circumference, and calf circumference. The estimate formulas for waist circumference were developed using simple and multiple regression analysis. Results: Simple regression analysis indicated that body mass index, upper arm circumference, forearm circumference, thigh circumference, and calf circumference were independent explanators for waist circumference ( $p<0.05$ for all). In addition, body mass index, upper arm circumference, and forearm circumference but not thigh circumference and calf circumference were extracted as independent explanators for waist circumference in multiple regression analysis $(p<0.05)$. We were able to develop the estimate formulas using body mass index, upper arm circumference, forearm circumference, thigh circumference, and calf circumference. Conclusion: The results suggest that the estimate formulas for waist circumference may provide an opportunity to easily evaluate waist circumference, even in hospitalized older adults with kyphosis posture. However, future studies should be conducted to develop the estimate formulas for waist circumference with a lower error value.

Keywords: Estimate formula, Waist circumference, Body mass index, Limb circumference, Hospitalized older adults

*Correspondence to Author:

Daisuke Takagi

Department of Physical Therapy, Health Science University: 7187 Kodachi Fujikawaguchiko-Town, Yamanashi, 401-0380, Japan.

TEL: +81 555-83-5295

How to cite this article:

Daisuke Takagi, Masatoshi Kageyama, Kenta Yamamoto, Hiroshi Matsumoto. Development of Estimate Formulas for Waist Circumference Using Body Mass Index and Limb Circumferences in Hospitalized Older Adults. International Journal of Aging Research, 2021, 4:76.

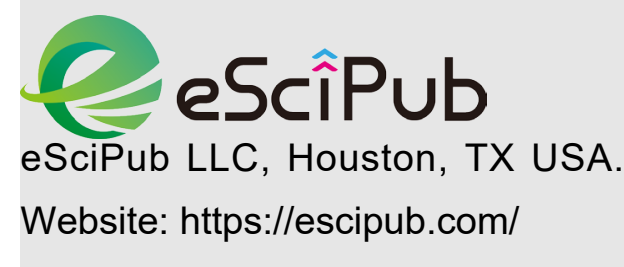




\section{Introduction}

According to the World Health Organization's 2016 report, ischemic heart disease and stroke are the main causes of death worldwide [1]. Metabolic syndrome results in an increased risk of onset of cardiovascular diseases and waist circumference (WC) as visceral fat is a required parameter for the diagnosis of metabolic syndrome in Japan ${ }^{[2,3]}$. WC has also been used as one measure of sarcopenic obesity related to metabolic syndrome ${ }^{[4,5]}$. Moreover, WC is positively associated with the homeostasis model assessment of insulin resistance (HOMA$\mathrm{IR}$ ), and patients with lacunar infarction and atherothrombotic infarction have been shown to have higher insulin resistance ${ }^{[6,7]}$. Thus, it is important to evaluate WC to prevent the emergence of ischemic heart disease or stroke. As compared with younger individuals, a higher proportion of individuals aged 60-69 and older than 70 years have a stronger suspicion of metabolic syndrome and being likely to suffer from metabolic syndrome [2]. As kyphosis posture is a characteristic of old age ${ }^{[8]}$, we often experience the inability to measure WC in older adults.

Body mass index (BMI) is an index of obesity and is calculated as the individual's body weight in kilograms divided by the square of the height in meters ${ }^{[2]}$. BMI can be easily measured in the clinical setting because it requires the uses of only height and weight. Circumference is also a simple measurement that requires only a tape measure. Circumference values reflect both muscle mass and fat mass ${ }^{[9-11]}$. Previous studies have reported that both BMI and circumferences at the upper and lower limbs are related to WC [12-14]. The estimate formula is a useful method for predicting WC without the dependence of posture. However, there have been few studies on the estimate formulas for WC using BMI or limb circumferences, which can be easily measured in older adults. Clarification of the estimate formulas using BMI and $\operatorname{limb}$ circumferences could provide an opportunity to use the values of WC in older adults and may consequently decrease the onset of ischemic heart disease or stroke.

We conducted the present investigation to develop estimate formulas of WC using BMI and limb circumferences in hospitalized older adults. We hypothesized that estimate formulas of WC using BMI and limb circumferences exist in hospitalized older adults.

\section{Materials and Methods}

\section{Participants}

For this cross-sectional study, we recruited 40 hospitalized older patients from Toyoda Eisei Hospital (average age \pm standard deviation [SD]: $81.4 \pm 7.4$; range, 69-97 years). Exclusion criteria included 1) the inability to obtain consent and 2) incomplete measurements. The average duration from the onset of major diseases was $31.1 \pm 31.6$ days. Major diseases represented in the sample included cardiovascular diseases (stroke: $22.5 \%$ [n = 9/40]; total $22.5 \%$ ), orthopedic diseases (femur fracture: $40.0 \%$ [n = 16/40]; vertebral fracture: $5.0 \%[n=2 / 40]$; patella or leg fracture: $5 \%[n=2 / 40]$; pelvic fracture: $7.5 \%[n=3 / 40]$; cervical fracture: $5.0 \%$ 
[n $=2 / 40]$; total $62.5 \%$ ], and others (disuse syndrome: $10.0 \%$ [n = 4/40], Guillain-Barre syndrome: $2.5 \%[n=1 / 40]$; Parkinson syndrome: $2.5 \%[n=1 / 40]$; total $15.0 \%$ ), respectively. The average Barthel index $(\mathrm{BI})$ score, which indicates the ability to carry out activities of daily living (ADL), ${ }^{[15]}$ is $58.8 \pm 18.1$ points. Total score in $\mathrm{BI}$ is ranged from 0 to 100 points and the lower point values show more need for assistance in ADL ${ }^{[15]}$. All participants read and signed an informed consent form, and this study was approved by the ethics committee of the Health Science University.

\section{BMI and WC}

BMI was calculated as the individual's body weight in kilograms divided by the square of the height in meters. WC was measured at the umbilicus with the subject in a standing posture at the end of mild expiration and was estimated to the nearest $0.1 \mathrm{~cm}$ using a tape measure. The average of two values of WC was analyzed as a representative value. The high reliability of WC has been observed, and intraclass correlation coefficients are greater than $0.900^{[16-18]}$.

\section{Limb circumference}

Upper arm circumference (UC), forearm circumference (FC), thigh circumference (TC), and calf circumference (CC) were measured at the largest points to the nearest $0.1 \mathrm{~cm}$. The measurements of each circumference were conducted twice using a tape measure. The intraobserver reliability of the circumference measurements ranged from good to perfect (0.65-0.99) [19]. UC and FC measurements were carried out in a sitting position when the participant's upper limbs were placed at the side of their body with their hands in a neutral position. For the TC values, participants were measured from a point $10 \mathrm{~cm}$ from the patella on the edge with the knee joint extension in the supine position. For CC measurements, participants were in a seated position with an approximate $90^{\circ}$ flexion of the hip and knee joints. Higher values of each of the average values of both the right and left sides were used for the analysis.

\section{Statistical analysis}

Data were expressed as means \pm SD. Statistical analysis was conducted using JMP 11 software (SAS Institute Inc., Cary, NC, USA). Relationships among age, height, weight, BMI, WC, UC, FC, TC, and CC were evaluated using Pearson's product- moment correlation coefficient (both parametric data) or Spearman's rank-correlation coefficient (either or both nonparametric data) according to the Shapiro-Wilk test. Differences in WC values between men and women were assessed using an unpaired $t$ test. In addition, we used simple or multiple regression analysis to develop the estimate formulas for predicting WC. Statistical significance was set at $p<0.05$.

\section{Results}

Table 1 presents the characteristics of the study patients. Using the Shapiro-Wilk test, age, weight, BMI, WC, UC, FC, TC, and CC were normally distributed, except for height. WC was significantly associated with height, weight, BMI, UC, FC, TC, and CC according to the Pearson' $s$ product-moment correlation coefficient or Spearman's rank-correlation coefficient, 
respectively (age: $r=-0.31, p=0.055$; height: $r$ shows the values of the correlation coefficients $=0.35, p=0.026$; weight: $r=0.78, p<.0001 ;$ with other items. Moreover, no significant BMI: $r=0.71, p<.0001$; UC: $r=0.82, p<.0001$; difference was observed in WC values between FC: $r=0.62, p<.0001$; TC: $r=0.66, p<.0001$; sexes using an unpaired $t$ test (both parametric CC: $r=0.58, p<.0001$; Table 2). Table 2 also data, $p=0.65$ ).

Table 1. Characteristics of the patients in this study

\begin{tabular}{ll}
\hline Characteristics $(\mathrm{n}=40)$ & \\
\hline Sex $(\mathrm{n}$, male / female) & $21 / 19$ \\
Age (years) & $81.4 \pm 7.4$ \\
Height $(\mathrm{cm})$ & $150.2 \pm 10.8$ \\
Weight $(\mathrm{kg})$ & $47.6 \pm 9.8$ \\
BMl $\left(\mathrm{kg} / \mathrm{m}^{2}\right)$ & $21.1 \pm 3.1$ \\
WC $(\mathrm{cm})$ & $82.1 \pm 8.7$ \\
UC $(\mathrm{cm})$ & $24.2 \pm 3.4$ \\
FC $(\mathrm{cm})$ & $21.5 \pm 2.5$ \\
TC $(\mathrm{cm})$ & $36.6 \pm 4.6$ \\
CC $(\mathrm{cm})$ & $30.3 \pm 3.9$ \\
\hline
\end{tabular}

Values were expressed mean \pm standard deviation; BMI, body mass index; WC, waist circumference; UC, upper arm circumference; FC, forearm circumference; TC, thigh circumference; CC, calf circumference.

BMI, UC, FC, TC, and CC were used to develop the estimate formulas for predicting WC. Simple regression analysis identified $\mathrm{BMI}, \mathrm{UC}, \mathrm{FC}, \mathrm{TC}$, and $C C$ as significant independent explanators for WC, respectively (BMI: $\beta=0.71$; UC: $\beta=$ 0.82; FC: $\beta=0.62$; TC: $\beta=0.66$; CC: $\beta=0.58$, all $p<.0001$ ). For BMI and UC, and for BMI and $\mathrm{FC}$ as predictor variables by multiple regression analysis, BMI, UC, and FC were all extracted as significant independent explanators for WC (BMI and UC BMI: $\beta=0.27, p=0.032$; UC: $\beta=0.64$, $p<.0001$; BMI and FC BMI: $\beta=0.54, p<.0001$; FC: $\beta=0.35, p=0.0054$ ) but not TC and CC in
$\mathrm{BMI}$ and $\mathrm{TC}$, and $\mathrm{BMI}$ and $\mathrm{CC}$ (BMI and TC BMI: $\beta=0.52, p=0.014 ; \mathrm{TC}: \beta=0.23, p=0.253$; BMI and CC BMI: $\beta=0.65, p=0.0009$; CC: $\beta=0.08$, $p=0.643$ ). Table 3 shows the estimate formulas for WC in the simple and multiple regression analyses. Furthermore, $R^{2}$ (coefficient of determination) and $\beta$ of the regression of the measured WC and estimated WC using the estimate formulas of UC, FC, TC, CC, BMl; BMI and $\mathrm{UC}$; $\mathrm{BMI}$ and $\mathrm{FC}$; $\mathrm{BMI}$ and $\mathrm{TC}$; and $\mathrm{BMI}$ and CC were 0.68 and 0.83 (UC), 0.38 and 0.62 (FC), 0.44 and 0.66 (TC), 0.34 and 0.58 (CC), 0.51 and $0.71(\mathrm{BMI}), 0.72$ and $0.85(\mathrm{BMI}$ and $\mathrm{UC})$, 
0.60 and 0.78 (BMI and FC), 0.53 and 073 (BMI respectively $(p<.0001$; Figure 1-1, 1-2, 1-3, 1-4, and $\mathrm{TC}$ ), and 0.51 and 0.71 (BMI and $\mathrm{CC}$ ), 1-5, 1-6, 1-7, 1-8, 1-9).

Table 2. Correlation coefficients among age, height, weight, BMI, WC, and limb circumference $(n=40)$

\begin{tabular}{|c|c|c|c|c|c|c|c|c|}
\hline & Age & Height & Weight & BMI & WC & UC & FC & $\mathrm{TC}$ \\
\hline Height & -0.27 & & & & & & & \\
\hline Weight & -0.29 & $0.65^{\star *}$ & & & & & & \\
\hline BMI & -0.10 & -0.03 & $0.73^{\star *}$ & & & & & \\
\hline WC & -0.31 & $0.35^{\star}$ & $0.78^{\star *}$ & $0.71^{\star *}$ & & & & \\
\hline UC & $-0.39^{*}$ & $0.38^{*}$ & $0.81^{\star *}$ & $0.69^{\star *}$ & $0.82^{* *}$ & & & \\
\hline FC & -0.28 & $0.69^{* *}$ & $0.86^{* *}$ & $0.49^{* \star}$ & $0.62^{\star *}$ & $0.69^{\star *}$ & & \\
\hline TC & -0.11 & 0.17 & $0.76^{* *}$ & $0.83^{* *}$ & $0.66^{\star *}$ & $0.71^{* *}$ & $0.55^{\star *}$ & \\
\hline CC & -0.02 & 0.15 & $0.69^{* *}$ & $0.77^{\star \star}$ & $0.58^{\star \star}$ & $0.61^{\star \star}$ & $0.52^{\star \star}$ & $0.80^{* *}$ \\
\hline
\end{tabular}

${ }^{*} p<0.05{ }^{* *} p<0.01$. Relationship between height and age, weight, BMI, WC, UC, FC, TC, and CC were evaluated using Spearman's rank-correlation coefficient and others with Pearson's product-moment correlation coefficient. BMI, body mass index; WC, waist circumference; UC, upper arm circumference; FC, forearm circumference; TC, thigh circumference; CC, calf circumference.

Table 3. Estimate formulas of WC using BMI, UC, FC, TC, and CC $(n=40)$

\begin{tabular}{lllc}
\hline Model & Estimate formulas & $\mathrm{R}^{2}$ & $\mathrm{SDE}$ \\
\hline 1 & $\mathrm{WC}=42.303+1.314 \times \mathrm{CC}$ & 0.34 & 7.2 \\
2 & $\mathrm{WC}=35.241+2.180 \times \mathrm{FC}$ & 0.38 & 7.0 \\
3 & $\mathrm{WC}=35.897+1.262 \times \mathrm{TC}$ & 0.44 & 6.6 \\
4 & $\mathrm{WC}=40.436+1.979 \times \mathrm{BMI}$ & 0.51 & 6.2 \\
5 & $\mathrm{WC}=31.592+2.088 \times \mathrm{UC}$ & 0.68 & 5.0 \\
6 & $\mathrm{WC}=38.450+1.801 \times \mathrm{BMI}+0.189 \times \mathrm{CC}$ & 0.51 & 6.3 \\
7 & $\mathrm{WC}=35.438+1.444 \times \mathrm{BMI}+0.444 \times \mathrm{TC}$ & 0.53 & 6.2 \\
8 & $\mathrm{WC}=23.935+1.495 \times \mathrm{BMI}+1.242 \times \mathrm{FC}$ & 0.60 & 5.7 \\
9 & $\mathrm{WC}=27.287+0.754 \times \mathrm{BMI}+1.610 \times \mathrm{UC}$ & 0.72 & 4.8 \\
\hline
\end{tabular}

$\mathrm{SDE}$, standard deviation of error; $R^{2}$, coefficient of determination. Units are given as $\mathrm{cm}$ for $\mathrm{WC}, \mathrm{kg} / \mathrm{m}^{2}$ for $\mathrm{BMI}, \mathrm{cm}$ for UC, $\mathrm{cm}$ for FC, cm for TC, cm for CC. BMI, body mass index; WC, waist circumference; UC, upper arm circumference; FC, forearm circumference; TC, thigh circumference; CC, calf circumference. 


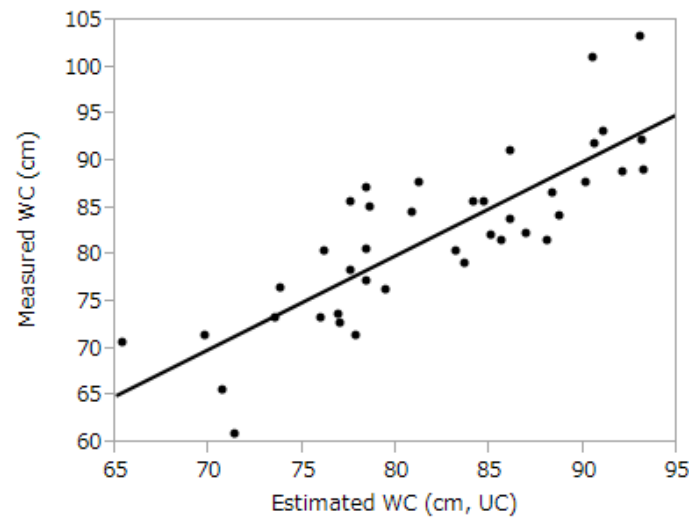

Figure 1-1. Measured WC and estimated WC (UC)

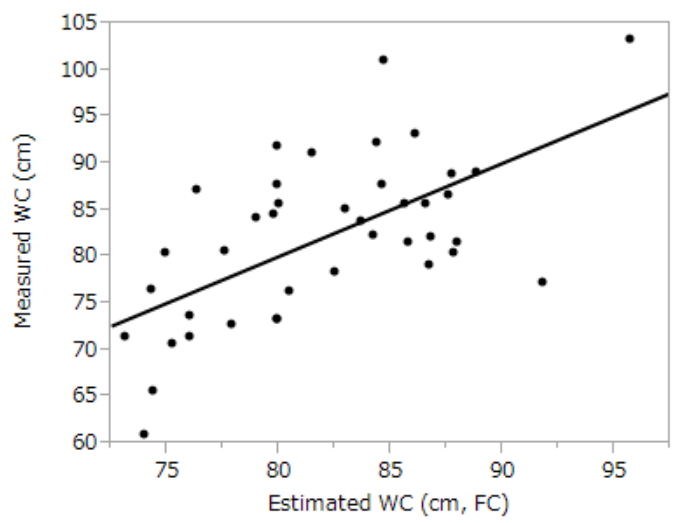

Figure 1-2. Measured WC and estimated WC (FC)

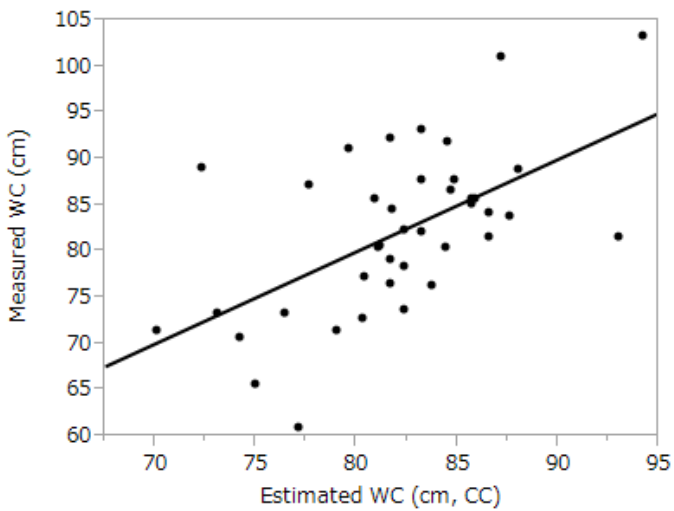

Figure 1-4. Measured WC and estimated WC (CC)

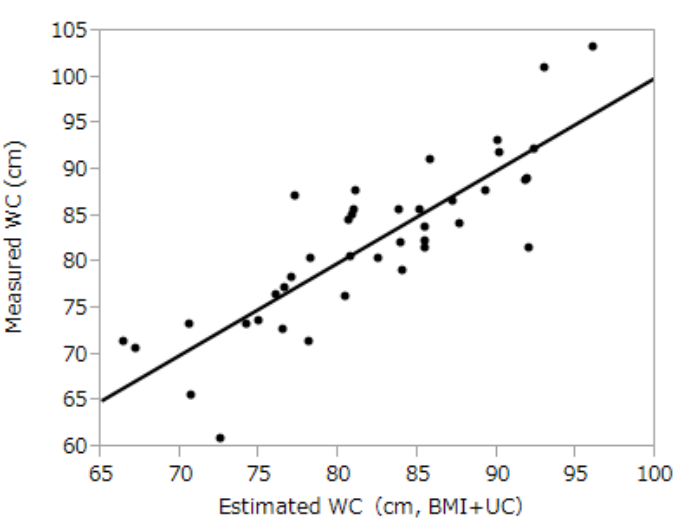

Figure 1-6. Measured WC and estimated WC (BMI+UC)

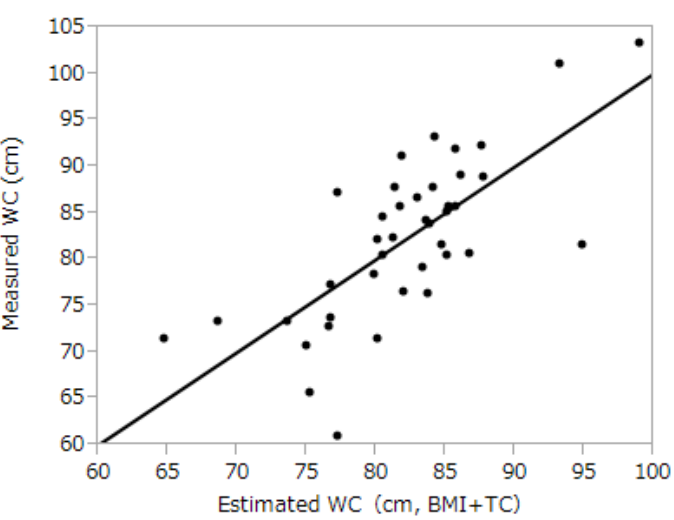

Figure 1-7. Measured WC and estimated WC (BMI+FC) Figure 1-8. Measured WC and estimated WC (BMI+TC) 


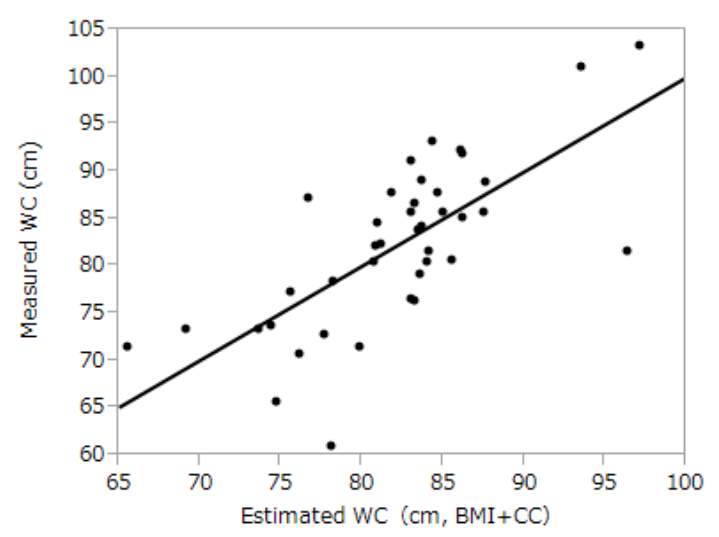

Figure 1-9. Measured WC and estimated WC $(B M I+C C)$

Figure 1-1, 1-2, 1-3, 1-4, 1-5, 1-6, 1-7, 1-8, 1-9. Relationship between the measured WC and estimated WC by estimate formulas. BMI, body mass index; WC, waist circumference; UC, upper arm circumference; FC, forearm circumference; TC, thigh circumference; CC, calf circumference.

\section{Discussion}

In this study, we found that WC was significantly associated with height, weight, BMI, UC, FC, TC, and $\mathrm{CC}$. BMI, UC, and $\mathrm{FC}$ were also independent explanators for $\mathrm{WC}$ in multiple regression analysis. We were able to develop the estimate formulas of WC using BMI, UC, FC, TC, and CC in hospitalized older adults. The results suggest that the estimate formulas may provide an opportunity to use the values of the WC in hospitalized older adults.

We observed significant correlations between WC and BMI, UC, FC, TC, and CC. A previous study reported that $W C$ is related to $B M I$ and the circumferences of the upper and lower limbs ${ }^{112-}$ 14]. In addition, BMI, UC, and FC were extracted as independent explanators for WC but not for $\mathrm{TC}$ or $\mathrm{CC}$ in the multiple regression analysis. UC and $\mathrm{FC}$ are generally classified as upper bodies as with WC. Therefore, UC and FC might reflect WC more easily than TC and CC. Moreover, the circumference values reflect both muscle mass and fat mass ${ }^{[9-11]}$. During the aging process, the muscle mass of the lower body tends to decrease compared with that of other parts of the body, and the antigravity muscles are easily influenced by immobilization ${ }^{[20-22]}$. Thus, TC and $\mathrm{CC}$ in $\mathrm{BMI}$ and $\mathrm{TC}$, and $\mathrm{BMI}$ and $\mathrm{CC}$ in the multiple regression analysis may not be independent explanators of WC in this study. Furthermore, the estimate formula using BMI and UC has the highest values of $R^{2}$, which is an index used to evaluate the suitability of multiple regression analysis. It has been reported that the mid upper arm circumference is associated with WC as central obesity [23]. Therefore, the estimate formula using BMI and UC may have the highest values of suitability from the multiple regression analysis in this study.

Typically, for estimate formulas, $R^{2}$ values greater than 0.5 are desirable. In this study, the calculated $R^{2}$ values for each of the estimate 
formulas ranged from 0.34 to 0.72 (Table 3 ), and the values for the estimate formulas using BMI, $\mathrm{UC}, \mathrm{BMI}$ and $\mathrm{UC}, \mathrm{BMI}$ and $\mathrm{FC}, \mathrm{BMI}$ and TC, and $\mathrm{BMI}$ and $\mathrm{CC}$ could be greater than 0.5 . On the other hand, in this study, the standard deviation of the error indicates that the error of the estimated WCs was $\pm 4.8-7.2 \mathrm{~cm}$. Thus, to use the WC predicted the estimate formulas of this study should be considered the errors, and future studies should develop the estimate formulas with a lower error value.

This study has some limitations. This research included only a small number of participants, and future studies should include a greater sample size. Moreover, we did not clarify the reasons for the error in the multiple regression analysis. Future studies should develop estimate formulas for WC using a lower standard deviation of error by clarifying the reasons for the error.

\section{Abbreviations:}

$A D L$, Activities of daily living

$\mathrm{BI}$, Barthel index

BMI, Body mass index

HOMA-IR, Homeostasis model assessment of insulin resistance

$\mathrm{SD}$, Standard deviation

UC, Upper arm circumference

FC, Forearm circumference

TC, Thigh circumference

$\mathrm{CC}$, Calf circumference

WC, Waist circumference

\section{References}

[1]. World Health Organization. The top causes of death. 2018. https://www.who.int/newsroom/fact-sheets/detail/the-top-10-causes-ofdeath (Accessed 20 November 2020).

[2]. Japanese society for the study of obesity. Guidelines for the management of obesity disease 2016,Life science, 2016, pp 4-8, 71 77 (in japanese).

[3]. Ninomiya T, Kubo M, Doi Y, Yonemoto K, Tanizaki $Y$ et al. Impact of metabolic syndrome on the development of cardiovascular disease in a general Japanese population: the Hisayama study. Stroke, 2007; 38(7): 2063-2069.

[4]. Lu CW, Yang KC, Chang HH, Lee LT, Chen CY et al. Sarcopenic obesity is closely associated with metabolic syndrome. Obes Res Clin Pract, 2013;7(4): e301-307.

[5]. Lee DC, Shook RP, Drenowatz C, Blair SN. Physical activity and sarcopenic obesity: definition, assessment, prevalence and mechanism. Future Sci OA, 2016;2(3): FSO127.

[6]. Sakurai T, limuro S, Araki A, Umegaki $H$, Ohashi $Y$ et al. Age-associated increase in abdominal obesity and insulin resistance, and usefulness of AHA/NHLBI definition of metabolic syndrome for predicting cardiovascular disease in Japanese elderly with type 2 diabetes mellitus. Gerontology, 2010; 56(2): 141-149.

[7]. Matsumoto K, Miyake S, Yano M, Ueki Y, Miyazaki A et al. Insulin resistance and classic risk factors in type 2 diabetic patients with different subtypes of ischemic stroke. Diabetes Care, 1999; 22(7): 1191-1195.

IJOAR: https://escipub.com/international-journal-of-aging-research/ 
[8]. Kado DM, Prenovost K, Crandall C. Narrative review: hyperkyphosis in older persons. Ann Intern Med, 2007; 147(5): 330-338.

[9]. Takagi D. Relationships among $\operatorname{limb}$ circumferences and appendicular muscle and fat masses using bioelectrical impedance analysis. Int J Phys Ther Rehab, 2018; 4: 148.

[10]. Rolland $\mathrm{Y}$, Lauwers-Cances V, Cournot $\mathrm{M}$, Nourhashémi F, Reynish W et al. Sarcopenia, calf circumference, and physical function of elderly women: a cross-sectional study. J Am Geriatr Soc, 2003; 51(8): 1120-1124.

[11]. Rolland Y, Czerwinski S, Abellan Van Kan G, Morley JE, Cesari M et al. Sarcopenia: its assessment, etiology, pathogenesis, consequences and future perspectives. J Nutr Health Aging,2008; 12(7): 433-450.

[12]. Katzmarzyk PT, Mire E, Bray GA, Greenway FL, Heymsfield SB et al. Anthropometric markers of obesity and mortality in white and African American adults: the pennington center longitudinal study. Obesity (Silver Spring). 2013; 21(5): 1070-1075.

[13]. Mason C, Craig CL, Katzmarzyk PT. Influence of central and extremity circumferences on allcause mortality in men and women. Obesity (Silver Spring), 2008; 16(12): 2690-2695.

[14]. Shi J, Yang Z, Niu Y, Zhang W, Li X et al. Large mid-upper arm circumference is associated with metabolic syndrome in middle-aged and elderly individuals: a community-based study. BMC Endocr Disord, 2020;20(1): 78.

[15]. Mahoney FI, Barthel DW. Functional evaluation: the barthel Index. Md State Med J, 1965; 14:61-65.

[16]. Nordhamn K, Södergren E, Olsson E, Karlstrom B, Vessby B et al. Reliability of anthropometric measurements in overweight and lean subjects: consequences for correlations between anthropometric and other variables. Int J Obes, 2000; 24(5): 652657.

[17]. Chen MM, Lear SA, Gao M, Frohlich JJ, Birmingham CL. Intraobserver and interobserver reliability of waist circumference and the waist-to-hip ratio. Obes Res, 2001; 9: 651.

[18]. Androutsos O, Anastasiou C, Lambrinou CP, Mavrogianni C, Cardon G et al. Intra- and inter- observer reliability of anthropometric measurements and blood pressure in primary schoolchildren and adults: the Feel4Diabetesstudy. BMC Endocr Disord, 2020; 20: 27.

[19]. Bakar Y, Özdemir ÖC, Sevim S, Duygu E, Tuğral $\mathrm{A}$ et al. Intra-observer and interobserver reliability of leg circumference measurement among six observers: a single blinded randomized trial. J Med Life, 2017; 10(3): 176-181.

[20]. Janssen I, Heymsfield SB, Wang ZM, Ross R. Skeletal muscle mass and distribution in 468 men and women aged 18-88 yr. J Appl Physiol (1985), 2000; 89(1): 81-88.

[21]. Tanimoto $\mathrm{Y}$, Watanabe M, Kono R, Hirota C, Takasaki $\mathrm{K}$ et al. Aging changes in muscle mass of Japanese. Nippon Ronen Igakkai Zasshi, 2010; 47(1): 52-57 (in japanese). 
[22]. Deitrick JE, Whedon GD, Shorr E. Effects of immobilization upon various metabolic and physiologic functions of normal men. Am J Med. 1948; 4(1): 3-36.

[23]. Zhu $Y$, Lin Q, Zhang Y, Deng H, Hu X et al. Mid-upper arm circumference as a simple tool for identifying central obesity and insulin resistance in type 2 diabetes. PLoS One, 2020; 15(5): e0231308. 